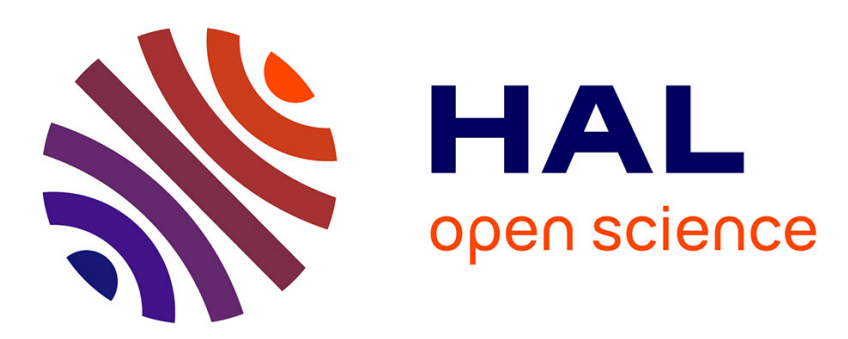

\title{
Analysis and Optimization of the Chemostat Model with a Lateral Diffusive Compartment
}

\author{
Maria Crespo, Alain Rapaport
}

\section{To cite this version:}

Maria Crespo, Alain Rapaport. Analysis and Optimization of the Chemostat Model with a Lateral Diffusive Compartment. Journal of Optimization Theory and Applications, 2020, 185, pp.597-621. 10.1007/s10957-020-01665-2 . hal-02513979

\section{HAL Id: hal-02513979 https://hal.science/hal-02513979}

Submitted on 21 Mar 2020

HAL is a multi-disciplinary open access archive for the deposit and dissemination of scientific research documents, whether they are published or not. The documents may come from teaching and research institutions in France or abroad, or from public or private research centers.
L'archive ouverte pluridisciplinaire HAL, est destinée au dépôt et à la diffusion de documents scientifiques de niveau recherche, publiés ou non, émanant des établissements d'enseignement et de recherche français ou étrangers, des laboratoires publics ou privés. 


\title{
Analysis and Optimization of the Chemostat Model with a Lateral Diffusive Compartment.
}

\author{
María Crespo • Alain Rapaport
}

Received: date / Accepted: date

\begin{abstract}
We consider the chemostat model with a side compartment connected by pure diffusion, and analyze its asymptotic properties. We investigate conditions under which this spatial structure is beneficial for species survival and conversion yield, compared to single chemostat. Under these conditions, we study the optimization problem for the best structure (volume distribution and diffusion rate), which minimizes the volume required to attain a desired conversion yield. The analysis reveals that particular configurations with a single tank connected by diffusion to the input stream can be the most efficient.
\end{abstract}

Keywords chemostat model $\cdot$ compartments $\cdot$ stability $\cdot$ conversion yield $\cdot$ optimal design

Mathematics Subject Classification (2000) 34D05 $\cdot 34 \mathrm{D} 23 \cdot 49 \mathrm{~K} 15 \cdot 92 \mathrm{E} 20$

\section{Introduction}

The model of the chemostat has been developed as a mathematical representation of the apparatus invented in the fifties simultaneously by Monod [1] and Novick \& Szilard [2], for studying the culture of micro-organisms. The chemostat setup consists of a microbial culture in a liquid media taking place in a vessel that is continuously fed with a nutritive substrate, while the media is removed with the same flow, so that the volume of the liquid in the vessel is kept constant. This way to cultivate micro-organisms is often called "continuous culture" as opposed to batch or María Crespo, Corresponding author

ETSII, Universidad Politécnica de Madrid

Madrid, Spain

maria.crespo@upm.es

Alain Rapaport,

MISTEA, Université Montpellier, INRAE, Montpellier SupAgro

Montpellier, France

alain.rapaport@inrae.fr 
sequential cultures, and is still today of prime importance (see for instance [3 5]). Its mathematical analysis has led to the so-called "theory of the chemostat" 6 10. This model is indeed widely used for industrial applications with continuously fed "bioreactors" for fermentation [11, 12] or waste-water treatment [4, 5, 13, but also in ecology for studying populations of micro-organisms (or plankton) in lakes, wetlands, rivers or aquaculture ecosystems 14 19. In all the above situations, a water resource is assumed to receive continuously an input flow with nutrients and to have an outlet of the same flow rate to keep its volume constant, justifying the application of the chemostat model although quite far from the original experimental setup. However, the classical model of the chemostat assumes a perfectly mixed media, which is often verified for small volumes. For industrial bioreactors or natural reservoirs with large volumes (such as lakes), the validity of this assumption becomes questionable. This is why several extensions of this model with spatial considerations have been proposed and studied in the literature.

The classical approaches for modeling non ideally mixed chemostats rely on a continuous representation of the spatial dimension (with systems of Partial Differential Equations, as in 20 22]) or on a finite number of interconnected compartments with different flow conditions in terms ofs systems of Ordinary Differential Equations, as in the "general gradostat" model $23[25]$ ). Most of the mathematical analysis available in the literature consider spatial heterogeneity only in the axial dimension of the bioreactors as in tubular or "plug-flow" bioreactors 26 30 and (simple) gradostats [31 34]. Surprisingly, configurations of tanks in parallel, rather than in series, have been much less investigated, apart from simple considerations in chemical reaction engineering 36,37 .

In many cases, the axial direction appears to be the one that generates the largest heterogeneity between the input and output (when the main current lines are along this axis), especially for high and relatively thin tanks under significantly large flow rate. From an operational view point, it is often reported that "dead zones" are observed in bioreactors and that the effective volumes of the tanks have to be corrected in the models to provide accurate predictions $36,38,43$. Segregated habitats are also considered in lakes, where the bottom can be modeled as a dead zone and nutrient mixing between the two zones is achieved by diffusion [44]. In a similar way, stagnant zones are well-known to occur in porous media such as soils, at various extents depending on soil structure. The effect of these dead zones on reactive and conservative mass transport, and in turn on the bio-geo-chemical cycles of elements, can be significant 45, 46. More generally, two-compartments models are used to describe ecological situations with two environments or two "patches" 47, 49]. The wording "dead-zone" might be slightly misleading as it can make believe that a part of the reservoir (where there is no advection stream) has no biological activity. But this does not necessarily mean that these "dead-zones" are entirely disconnected from other parts of the reactor. It is likely to be influenced by diffusion rather than convection. This is why we prefer to qualify these zones as "lateral-diffusive compartments".

The aim of the present work is to analyze the chemostat model with two compartments (or two vessels), one of them being connected by "lateral-diffusion", and to investigate conditions under which having this additional compartment could be beneficial for the conversion yield compared to the classical chemostat model with a single compartment 
of the same total volume. This structure is sometimes called side-capacity or cross-flow model (see, e.g., 50 52]) and it can be seen as a particular case of the general gradostat. Historically, the gradostat has been invented as an experimental device to mimic spatial heterogeneity in continuous culture [31,32, and consists in tanks of identical volumes connected in series and subject to the same dilution rate, which has led to the mathematical theory of the gradostat 33, 34. Later, this model has been generalized to the so-called "general gradostat" model which considers more general networks of interconnected tanks with possible different volumes and dilution rates $[7,23$. Nevertheless, those results are too general to give explicit conditions on the parameters for the existence of a positive equilibrium and its stability, and do not compare the performances of each configuration, which is the main purpose of the present work. The structure considered here can be also seen as a limiting case of the pattern "chemostats in parallel with diffusion connection" studied in [25], with only one vessel receiving an input flow rate. Nevertheless, this later reference imposes some restrictions, such as linear reaction between species and removal rate large enough to avoid washout with a single tank. In the present work, we conduct a deeper model analysis and investigate the effects of lateral diffusion from two view points.

1. In terms of ecological impact, we study the effect of the diffusion on a given volume distribution regarding the conversion yield. In particular, we aim at characterizing situations for which, having a structure with lateral diffusion is better than having a single perfectly mixed volume.

2. In terms of economic benefits, we look for the best volume distribution which, for a given diffusion rate, minimizes the total volume required to attain a desired level of conversion. This allows us to revisit the optimal design problem with such configurations, that was previously tackled but considering tanks connected in series (see, e.g., [53 55]). Finally, we aim at determining the diffusion rate parameter that gives the best volume reduction.

In practice, the diffusion rate parameter can be estimated with the help of inert tracers [35] and depends on the shape of the reservoir relatively to the advective direction [36] (a thick reservoir is expected to have more lateral dispersion than a thin one). The design of industrial reactors is a combination of static factors (shapes, interconnections...) and dynamical considerations (mixing conditions, recirculation loops...) that can be chosen to adjust a communication rate between tanks or zones of a reservoir 36,37. This is why we consider also the optimization with respect to this communication parameter.

The article is organized as follows. In Section 2 we introduce the model describing the dynamics of the chemostat composed of two compartments (one of them being connected by diffusion) and determine the steady states. We investigate the conversion yield (according to the diffusion rate parameter) and characterize when this structure is better than the single chemostat (i.e. a single perfectly mixed volume). Section 3 is then dedicated to optimal design questions, firstly when the diffusion rate is fixed, and then when it can be adjusted. Particularly, Sub-section 3.3 presents a numerical illustration of the profit that can be obtained considering a lateral vessel. Finally, Section 4 
discusses and interprets the results, providing graphical interpretations. With a view to shorten the presentation, the proofs of the preliminary results of Section 2 have been placed in the Appendix.

\section{Model Description and Equilibria Analysis}

We consider configurations of one chemostat of volume $V_{1}$ interconnected by Fickian diffusion with a tank of volume $V_{2}$, as depicted on Figure1. This kind of configurations can be seen as a particular case of the general gradostat with two compartments (see, e.g., 7]) or a parallel interconnection of two compartments (taking $\alpha=r=1$ ), as introduced in 25. Denoting by $s_{i}, x_{i}$ the concentrations of substrate and biomass in tank $i=1,2$, the model is written as follows

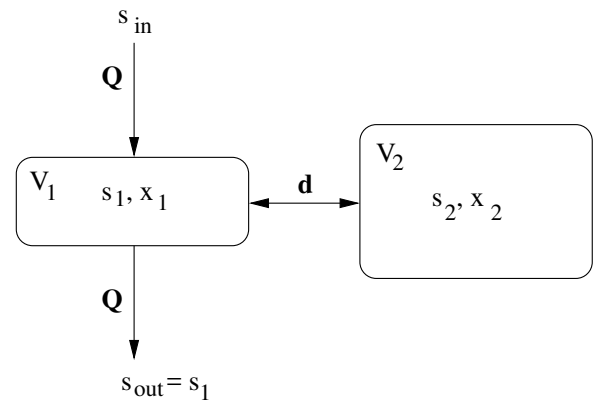

Fig. 1 Interconnection of a chemostat of volume $V_{1}$ with a lateral compartement of volume $V_{2}$.

$$
\left\{\begin{array}{l}
\dot{s}_{1}=-\mu\left(s_{1}\right) x_{1}+\frac{Q}{V_{1}}\left(s_{\text {in }}-s_{1}\right)+\frac{d}{V_{1}}\left(s_{2}-s_{1}\right) \\
\dot{x}_{1}=\mu\left(s_{1}\right) x_{1}-\frac{Q}{V_{1}} x_{1}+\frac{d}{V_{1}}\left(x_{2}-x_{1}\right) \\
\dot{s}_{2}=-\mu\left(s_{2}\right) x_{2}+\frac{d}{V_{2}}\left(s_{1}-s_{2}\right) \\
\dot{x}_{2}=\mu\left(s_{2}\right) x_{2}+\frac{d}{V_{2}}\left(x_{1}-x_{2}\right)
\end{array}\right.
$$

(we have assumed, without any loss of generality, that the stochiometry of transformation of substrate into biomass is equal to 1 , which can be always obtained by simply rescaling the biomass concentrations $x_{i}$ ). The parameters $Q$ and $s_{\text {in }}$ denote the flow rate and substrate concentration of the input stream, while the parameter $d>0$ is the diffusion coefficient between the two tanks Here, we assume that biomass is not prone to fix on the walls or aggregates into biofilms, so that we consider the diffusion rate to be identical for the substrate and the micro-organisms. The specific growth rate function of the micro-organisms is denoted $\mu$ and fulfills the usual assumption.

Hypothesis 1 The growth function $\mu(\cdot)$ is an increasing concave function with $\mu(0)=0$.

A typical such instance of function $\mu$ is given by the well-known Monod law (see, e.g., 7, 8]):

$$
\mu(s)=\mu_{\max } \frac{s}{K+s} .
$$


Notice that, in [25], the authors assume that the growth rate function is linear.

On Figure 1, $s_{\text {out }}=s_{1}$ represents the output concentration of substrate that comes out of the system with flow rate $Q$. Throughout the paper, we shall consider the "conversion yield" as the ratio $\left(s_{i n}-s_{\text {out }}\right) / s_{i n}$. For a given input concentration $s_{i n}$, the lower is $s_{\text {out }}$ the higher is the conversion yield.

It is convenient for the following to introduce the function

$$
\beta(s)=\mu(s)\left(s_{\mathrm{in}}-s\right)
$$

for which one one can easily check the following property.

Lemma 2.1 Under Hypothesis 1 , the function $\beta$ is strictly concave on $\left[0, s_{\mathrm{in}}\right]$ and there exists a unique value

$$
\hat{s}=\arg \max _{s \in\left(0, s_{\text {in }}\right)} \beta(s) .
$$

The value $\beta(s)$ represents the steady-state growth rate of the micro-organisms when the substrate concentration is equal to $s$ at steady-state.

Let us start by some preliminary results, whose proofs can be found in the Appendix.

Lemma 2.2 The non-negative orthant $\mathbb{R}_{+}^{4}$ is invariant by dynamics (1) and any solution in $\mathbb{R}_{+}^{4}$ is bounded.

Although existence and stability of equilibria of interconnected chemostats have been studied in the literature in a general setup (see, e.g., $[7]$ ), we particularize here the condition for which the washout state $\mathbf{E}^{0}=\left(0, s_{\text {in }}, 0, s_{\text {in }}\right)^{\top}$ is the unique steady-state of the dynamics (1). For this purpose, we introduce the following polynomial:

$$
P(X)=V_{1} V_{2} X^{2}-\left(d V_{1}+(Q+d) V_{2}\right) X+d Q .
$$

Proposition 2.1 Under Hypothesis 1, one has

(i) The washout equilibrium $\mathbf{E}^{0}$ is the unique steady state of (1) exactly when $s_{\text {in }}$ satisfies the condition

$$
\mu\left(s_{\text {in }}\right) \leq \frac{Q}{V_{1}} \text { and } P\left(\mu\left(s_{\text {in }}\right)\right) \geq 0 .
$$

It is then globally asymptotically stable on $\mathbb{R}_{+}^{4}$. 
(ii) When condition (5) is not fulfilled, there exists an unique positive steady state $\mathbf{E}^{\star}$ of (1) distinct from $\mathbf{E}^{0}$. Then, for any initial condition except on a set of null measure, the solution of (1) converges asymptotically to $\mathbf{E}^{\star}$, which is moreover locally exponentially stable.

Let us underline that the condition (5) is more restrictive that the single condition $\mu\left(s_{\text {in }}\right) \leq \frac{Q}{V_{1}}$ (one can check that $P\left(\frac{Q}{V_{1}}\right)<0$ when $d>0$ ), which is the exact condition for wash-out in the single tank (i.e. $\left.d=0\right)$. Differently to serial configuration, the lateral connection is thus less prone to wash-out.

Now, we investigate how a second compartment influences the output concentration of the substrate, compared to a single tank configuration of the same total volume $V_{1}+V_{2}$. To this aim, we fix $V_{1}, V_{2}$ and $Q$, and study the output map $d \rightarrow s_{1}^{\star}(d)$ at steady state, as a function of the diffusion parameter $d$. The benefits of the structured chemostat in terms of conversion yield are discussed in Section 4. Proposition 2.1 gives implicitly the existence of the map $s_{1}^{\star}(\cdot)$ for the unique non-trivial steady-state of system (11), but it does not give explicit ranges of existence of this steady-state, depending on the operating parameters $Q$ and $s_{i n}$. This issue has not been addressed in former works, such as in [25], where it has been simply assumed that the flow rate was "large enough" to avoid washout with a single tank.

Proposition 2.2 Let $V=V_{1}+V_{2}$ and define the number

$$
\bar{d}=V_{2} \mu\left(s_{\text {in }}\right) \frac{Q-V_{1} \mu\left(s_{\text {in }}\right)}{Q-\left(V_{1}+V_{2}\right) \mu\left(s_{\text {in }}\right)} .
$$

It follows that:

(i) If $\mu\left(s_{\mathrm{in}}\right)<Q / V$, then the non-trivial equilibrium $s_{1}^{\star}(d)<s_{\text {in }}$ exists when $\left.d \in\right] 0, \bar{d}[$.

(ii) If $Q / V \leq \mu\left(s_{\text {in }}\right) \leq Q / V_{1}$, then the non-trivial equilibrium $s_{1}^{\star}(d)<s_{\text {in }}$ exists when $d>0$.

(iii) If $\mu\left(s_{\text {in }}\right)>Q / V_{1}$, then the non-trivial equilibrium $s_{1}^{\star}(d)<s_{\text {in }}$ exists when $d \geq 0$.

Proof. When $d=0$ (that is, when the lateral tank is detached), the classical equilibria analysis of the single chemostat model with volume $V_{1}$ (the reader may refer to e.g., [7,8]) ensures that the positive equilibrium $s_{1}^{\star}$ exists exactly when $\mu\left(s_{\text {in }}\right)>Q / V_{1}$, which corresponds to the case (iii) on the proposition statement.

When $d>0$, we prove cases (i)-(iii) by taking into account that it correspond to three different scenarios where condition (5) is not fulfilled. For ease of reasoning, we rewrite $P\left(\mu\left(s_{\mathrm{in}}\right)\right)$ as

$$
P\left(\mu\left(s_{\text {in }}\right)\right)=V_{2} \mu\left(s_{\text {in }}\right) \underbrace{\left(V_{1} \mu\left(s_{\text {in }}\right)-Q\right)}_{\Delta_{1}}+d \underbrace{\left(Q-\left(V_{1}+V_{2}\right) \mu\left(s_{\text {in }}\right)\right)}_{\Delta_{2}} .
$$

(i) In this case, the non-trivial equilibrium exists when $P\left(\mu\left(s_{\text {in }}\right)\right)<0$. Straightforwardly, one has $\Delta_{1}<0, \Delta_{2}>0$ and then $s_{1}^{\star}(d)<s_{\text {in }}$ exists when $0<d<\bar{d}=-V_{2} \mu\left(s_{\text {in }}\right) \Delta_{1} / \Delta_{2}$.

(ii) In this case, the non-trivial equilibrium exists when $P\left(\mu\left(s_{\text {in }}\right)\right)<0$. Straightforwardly, one has $\Delta_{1}<0, \Delta_{2}<0$ 
and then $s_{1}^{\star}(d)<s_{\text {in }}$ exists for any $d>0$.

(iii) In this case, the non-trivial equilibrium exists for any value of $P\left(\mu\left(s_{\text {in }}\right)\right)$, and then $s_{1}^{\star}(d)<s_{\text {in }}$ exists for any $d \geq 0$

Remark 2.1 One can easily check how the two extreme situations (no diffusion and infinite diffusion) are recovered:

(i) When $\mu\left(s_{\text {in }}\right)>Q / V_{1}$, the non trivial equilibrium of system $[1]$ satisfies $s_{1}^{\star}(0)=s_{1}^{\star, 0}$, where $s_{1}^{\star, 0}=\mu^{-1}\left(\frac{Q}{V_{1}}\right)$ is the non-trivial steady state of a single chemostat model with volume $V_{1}$. In other case one has $\lim _{d \rightarrow 0^{+}} s_{1}^{\star}(d)=s_{\text {in }}$.

(ii) When $\mu\left(s_{\text {in }}\right) \geq Q / V$, the non trivial equilibrium of system 11 verifies $\lim _{d \rightarrow+\infty} s_{1}^{\star}(d)=s_{1}^{\star, \infty}$, where $s_{1}^{\star, \infty}=$ $\mu^{-1}\left(\frac{Q}{V}\right)$ is the non-trivial steady state of the single chemostat model with volume $V=V_{1}+V_{2}$.

Proposition 2.3 Let $\hat{s}$ be defined in (4) and $V=V_{1}+V_{2}$. It follows that:

(i) If $\mu\left(s_{\mathrm{in}}\right)<Q / V$, then the map $d \rightarrow s_{1}^{\star}(d)$ admits a minimum at $d^{\star}<\bar{d}$, that is strictly less than $s_{\mathrm{in}}$.

(ii) If $\mu\left(s_{\mathrm{in}}\right) \geq Q / V$ and $s_{1}^{\star, \infty}<\hat{s}$, then the map $d \rightarrow s_{1}^{\star}(d)$ admits a minimum in $d^{\star}<+\infty$, that is strictly less than $s_{1}^{\star, \infty}$.

(iii) If $\mu\left(s_{\mathrm{in}}\right) \geq Q / V$ and $s_{1}^{\star, \infty} \geq \hat{s}$, then the map $d \rightarrow s_{1}^{\star}(d)$ is decreasing and $s_{1}^{\star}(d)>s_{1}^{\star, \infty}$ for any $d>0$.

Proof. $\mathbf{s}^{\star}=\left(s_{1}^{\star}, s_{2}^{\star}\right) \in\left(0, s_{\text {in }}\right) \times\left(0, s_{\text {in }}\right)$ is solution of

$$
\left\{\begin{array}{l}
d\left(s_{2}^{\star}-s_{1}^{\star}\right)=\left(V_{1} \mu\left(s_{1}^{\star}\right)-Q\right)\left(s_{\text {in }}-s_{1}^{\star}\right), \\
d\left(s_{1}^{\star}-s_{2}^{\star}\right)=V_{2} \mu\left(s_{2}^{\star}\right)\left(s_{\text {in }}-s_{2}^{\star}\right) .
\end{array}\right.
$$

If one differentiates system (7) with respect to $d$, it follows that

$$
\begin{gathered}
\left(s_{2}^{\star}-s_{1}^{\star}\right)+d\left(\partial_{d} s_{2}^{\star}-\partial_{d} s_{1}^{\star}\right)=\partial_{d} s_{1}^{\star} \underbrace{\left(Q+V_{1} \mu^{\prime}\left(s_{1}^{\star}\right)\left(s_{\text {in }}-s_{1}^{\star}\right)-V_{1} \mu\left(s_{1}^{\star}\right)\right)}_{\Omega_{1}}, \\
\left(s_{1}^{\star}-s_{2}^{\star}\right)+d\left(\partial_{d} s_{1}^{\star}-\partial_{d} s_{2}^{\star}\right)=\partial_{d} s_{2}^{\star} \underbrace{\left(V_{2} \mu^{\prime}\left(s_{2}^{\star}\right)\left(s_{\text {in }}-s_{2}^{\star}\right)-V_{2} \mu\left(s_{2}^{\star}\right)\right)}_{\Omega_{2}},
\end{gathered}
$$

which can be rewritten as

$$
\underbrace{\left[\begin{array}{cc}
\Omega_{1}+d & -d \\
d & -\Omega_{2}-d
\end{array}\right]}_{\Gamma}\left(\begin{array}{l}
\partial_{d} s_{1}^{\star} \\
\partial_{d} s_{2}^{\star}
\end{array}\right)=\left(s_{2}^{\star}-s_{1}^{\star}\right)\left(\begin{array}{l}
1 \\
1
\end{array}\right) .
$$

Remark that one has

$$
\Omega_{1}+d=d \phi_{1}^{\prime}\left(s_{1}^{\star}\right), \quad \Omega_{2}+d=d \phi_{2}^{\prime}\left(s_{2}^{\star}\right), \quad \operatorname{det}(\boldsymbol{\Gamma})=d^{2}\left(1-\phi_{1}^{\prime}\left(s_{1}^{\star}\right) \phi_{2}^{\prime}\left(s_{2}^{\star}\right)\right) .
$$


It can be proved that one has necessarily $\operatorname{det}(\boldsymbol{\Gamma})<0$ (see the proof of Proposition 2.1 in the Appendix), and then the derivatives $\partial_{d} s_{1}^{\star}$ and $\partial_{d} s_{2}^{\star}$ can be defined as

$$
\partial_{d} s_{1}^{\star}=\left(s_{2}^{\star}-s_{1}^{\star}\right) \frac{-\Omega_{2}}{\operatorname{det}(\boldsymbol{\Gamma})}, \quad \partial_{d} s_{2}^{\star}=\left(s_{2}^{\star}-s_{1}^{\star}\right) \frac{\Omega_{1}}{\operatorname{det}(\boldsymbol{\Gamma})} .
$$

Let us first show that $\Omega_{1}>0$ by proving that $\phi_{1}^{\prime}\left(s_{1}^{\star}(d)\right)>1$. From Proposition 2.1, one has that the positive steady-state fulfills

$$
0<s_{1}^{\star}(d)<\lambda_{1}\left(s_{\text {in }}\right)=\min \left(s_{\text {in }}, s_{1}^{\star, 0}\right) .
$$

Since $\phi_{1}$ is concave (equivalently, $\phi_{1}^{\prime}$ is decreasing) on $\left[0, \lambda_{1}\left(s_{\text {in }}\right)\right]$, one has that $\phi_{1}^{\prime}\left(s_{1}^{\star}(d)\right)>\phi_{1}^{\prime}\left(\lambda_{1}\left(s_{\text {in }}\right)\right)$.

- If $\mu\left(s_{\text {in }}\right) \leq Q / V_{1}$, then $\lambda_{1}\left(s_{\text {in }}\right)=s_{\text {in }}$ and $\phi_{1}^{\prime}\left(s_{\text {in }}\right)=1+\frac{Q-V_{1} \mu\left(s_{\text {in }}\right)}{d}>1$.

- If $\mu\left(s_{\text {in }}\right)>Q / V_{1}$, then $\lambda_{1}\left(s_{\text {in }}\right)=s_{1}^{\star, 0}$ and $\phi_{1}^{\prime}\left(s_{1}^{\star, 0}\right)=1+\frac{V_{1}}{d} \mu^{\prime}\left(s_{1}^{\star, 0}\right)\left(s_{\text {in }}-s_{1}^{\star, 0}\right)>1$.

One has then $\phi_{1}^{\prime}\left(\lambda_{1}\left(s_{\text {in }}\right)\right)>1$ in any case, which proves that $\Omega_{1}>0$. Therefore one has $\partial_{d} s_{2}^{\star}>0$, i.e., $s_{2}^{\star}(\cdot)$ is an increasing map. Now, notice that $\Omega_{2}=V_{2} \beta^{\prime}\left(s_{2}^{\star}(d)\right)$ and its sign depends on the relative position of $s_{2}^{\star}(d)$ with respect to parameter $\hat{s}$.

(i) Since $s_{2}^{\star}(\cdot)$ is increasing, $\lim _{d \rightarrow 0} s_{2}^{\star}(d)=0, \lim _{d \rightarrow \bar{d}} s_{2}^{\star}(d)=s_{\text {in }}$ and $\hat{s} \in\left(0, s_{\text {in }}\right)$. By using the Mean Value Theorem, it follows that there exists a unique value $d \in] 0, \bar{d}\left[\left(\right.\right.$ denoted by $\left.d^{\star}\right)$ such that $s_{2}^{\star}\left(d^{\star}\right)=\hat{s}$, with $\beta^{\prime}\left(s_{2}^{\star}(d)\right)>0$ for $d<d^{\star}$ and $<0$ for $d>d^{\star}$. Consequently, $\partial_{d} s_{1}^{\star}$ admits a unique minimum in $d^{\star}$, as $\operatorname{sgn}\left(\partial_{d} s_{1}^{\star}(d)\right)=-\operatorname{sgn}\left(\Omega_{2}\right)$.

(ii) Since $s_{2}^{\star}(\cdot)$ is increasing, $\lim _{d \rightarrow 0} s_{2}^{\star}(d)=0, \lim _{d \rightarrow+\infty} s_{2}^{\star}(d)=s_{1}^{\star, \infty}$ and $\hat{s} \in\left(0, s_{1}^{\star, \infty}\right)$. By using the Mean Value Theorem, it follows that there exists a unique value $d>0$ (denoted by $\left.d^{\star}\right)$ such that $s_{2}^{\star}\left(d^{\star}\right)=\hat{s}$. Consequently, $\partial_{d} s_{1}^{\star}$ admits a unique minimum in $d^{\star}$, with $s_{1}^{\star}(\cdot)$ decreasing on $\left[0, d^{\star}[\right.$ and increasing on $] d^{\star},+\infty\left[\right.$. As $s_{1}^{\star}(\cdot)$ is increasing on $] d^{\star},+\infty\left[\right.$ and $\lim _{d \rightarrow+\infty} s_{1}^{\star}(d)=s_{1}^{\star, \infty}$ (see Remark 2.1], one necessarily has $s_{1}^{\star}\left(d^{\star}\right)<s_{1}^{\star, \infty}$.

(iii) Since $s_{2}^{\star}(\cdot)$ is increasing, $\lim _{d \rightarrow+\infty} s_{2}^{\star}(d)=s_{1}^{\star, \infty}$ and $\hat{s}>s_{1}^{\star, \infty}$, one has that $\beta^{\prime}\left(s_{2}^{\star}(d)\right)>0$, i.e., $s_{1}^{\star}(d)$ is decreasing for any $d>0$. As $\lim _{d \rightarrow+\infty} s_{1}^{\star}(d)=s_{1}^{\star, \infty}$, it follows that $s_{1}^{\star}(d)>s_{1}^{\star, \infty}$.

A schematic representation of the three possible situations given by Proposition 2.3 is depicted on Figure 2 As recalled in Remark 2.1, the limiting case $d=+\infty$ represents in these pictures the configuration of a single chemostat of volume $V$. In case (a), there is no positive equilibrium when $d$ is larger than $\bar{d}$. Then, the only possible steady-state is the wash-out (remind that one has $s_{1}=s_{i n}$ at wash-out). In cases (b) and (c) the output concentration for the single chemostat at non wash-out steady state is represented by the value $s_{1}^{\star, \infty}$. 


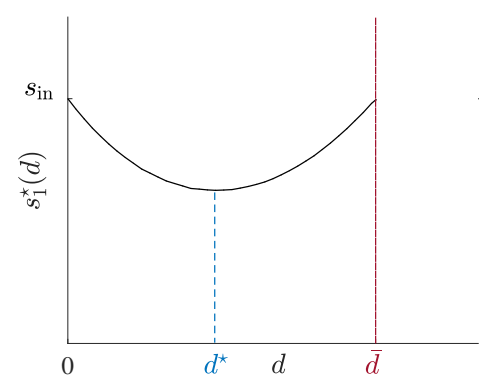

(a) $\mu\left(s_{\text {in }}\right)<\frac{Q}{V}$

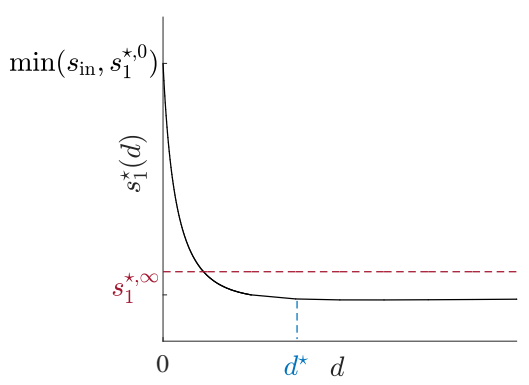

(b) $\mu\left(s_{\text {in }}\right) \geq Q / V$ and $s_{1}^{\star, \infty}<\hat{s}$

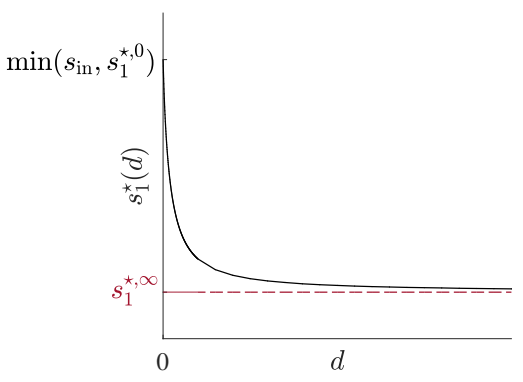

(c) $\mu\left(s_{\text {in }}\right) \geq Q / V$ and $s_{1}^{\star, \infty} \geq \hat{s}$

Fig. 2 Plot of the function $d \rightarrow s_{1}^{\star}(d)$ for the three situations depicted in Proposition 2.3

Cases (a) and (b) are situations for which spatial heterogeneity is beneficial for the conversion yield, while case (c) is always less efficient than a perfectly mixed volume. In case (a), there exists a positive steady-state for any $d$ between 0 and $\bar{d}$, which is therefore better than the wash-out of the single chemostat. In case (b), the concentration $s_{1}^{\star}$ at steady-state is lower than the single chemostat one, provided that the parameter $d$ is large enough, differently to case (a). Notice that case (a) corresponds to inputs that lead to the wash-out of a perfectly mixed reactor of volume $V$, which is usually not considered in the literature because it has no practical interest. However, we show here that a spatial heterogeneity could allow a substrate conversion, under such inputs conditions. In both cases (a) and (b), it can be seen that the output substrate concentration depends on parameter $d$ and has a minimum at some value $d^{*}$, for which the conversion yield improves the one obtained with a single tank of volume $V$. In contrast, when case (c) occurs, the output concentration systematically decreases with parameter $d$, and is always larger compared to a perfectly mixed volume $V$. This point will be discussed in Section 4

Remark 2.2 The limit case $V_{1}=0$ corresponds to a single tank (of volume $V_{2}$ ) connected by diffusion to the input pipe with flow rate $Q$ (see Figure 3). There is no biological activity in the pipe, but simply a dilution given by the

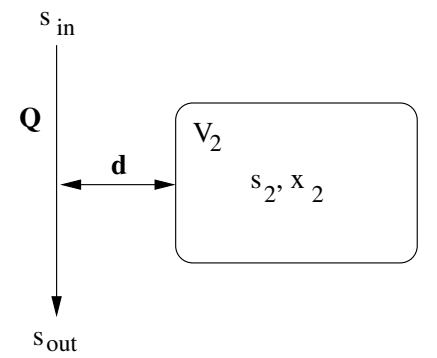

Fig. 3 The limiting case of $V_{1}=0$. Input charged with substrate at a concentration $s_{\text {in }}$ flows with rate $Q$ along a pipe. Due to diffusion, substrate reacts with biomass at the lateral tank and leaves the pipe with flow rate $Q$. 
mass balance at the connection point:

$$
\left\{\begin{array}{l}
Q\left(s_{\text {in }}-s_{\text {out }}\right)=d\left(s_{\text {out }}-s_{2}\right) \\
-Q x_{\text {out }}=d\left(x_{\text {out }}-x_{2}\right)
\end{array} \quad \Rightarrow \quad s_{\text {out }}=\frac{Q s_{\text {in }}+d s_{2}}{Q+d}, x_{\text {out }}=\frac{d x_{2}}{Q+d}\right.
$$

Then the dynamics in the tank with $\left(s_{\text {out }}, x_{\text {out }}\right)$, instead of $\left(s_{1}, x_{1}\right)$, is given by the equations

$$
\left\{\begin{array}{l}
\dot{s}_{2}=-\mu\left(s_{2}\right) x_{2}+\frac{Q d}{(Q+d) V_{2}}\left(s_{\text {in }}-s_{2}\right) \\
\dot{x}_{2}=\mu\left(s_{2}\right) x_{2}-\frac{Q d}{(Q+d) V_{2}} x_{2}
\end{array}\right.
$$

which is equivalent to have a single tank of volume $V_{2}$ with input flow rate $Q d /(Q+d)$ and output concentration $s_{\text {out }}=\left(Q s_{\text {in }}+d s_{2}\right) /(Q+d)$. We shall bear in mind that this configuration is playing a role in the optimization problem of the next section. The limit case $V_{2}=0$ corresponds to the chemostat model with one tank of volume $V_{1}$, or equivalently having no diffusion (i.e., $d=0$ ).

\section{Optimal Configurations}

In this section, we aim at characterizing the most efficient configurations, that are the best parameters of the structured chemostat depicted on Figure 1 (reactor volumes and diffusion rate) minimizing the total volume, the output substrate concentration being prescribed at steady state. Let us underline that this criterion is equivalent to minimizing the mean residence time in the process, which is commonly defined as

$$
\bar{t}=\frac{\int_{0}^{+\infty} t s_{\text {out }}(t) d t}{\int_{0}^{+\infty} s_{\text {out }}(t) d t}
$$

when at time 0 there is a uniform unitary concentration of the substrate (i.e. $s_{i}(0)=1$ for any $i$ ) with no biological activity and input concentration at any future time (i.e. $x(\cdot) \equiv 0$ and $s_{i n}=0$ ). One can easily show that $\bar{t}=V / Q$, where $V$ is the total volume, whatever is the spatial configuration. More details about the mean residence time can be found for instance in [51, Chapter 15].

This section is organized as follows. In Sub-section 3.1. we first tackle the problem when the diffusion parameter is fixed. Then, in Sub-section 3.2 we address the full optimization problem in which the diffusion parameter is also considered as an optimization variable. Interpretations of the optimal results are presented in Section 4 


\subsection{Parameter $\boldsymbol{d}$ is Fixed}

We target here situations for which the diffusion parameter $d$ is imposed by the hydrodynamics conditions between the two patches, and aim at characterizing the most efficient distribution of the volumes.

Given a nominal desired value $s_{\text {ref }}<s_{\text {in }}$ as output of the process, we look for solutions of the optimization problem

$$
\min _{\left(V_{1}, V_{2}\right) \in \mathbb{R}_{+}^{2}}\left\{V_{1}+V_{2}: \text { such that } s_{1}=s_{\text {ref }} \text { at steady state }\right\}
$$

that we denote by $\left(V_{1}^{\mathrm{opt}}, V_{2}^{\mathrm{opt}}\right)$.

For the analysis of the solution of problem $[10$, , it is convenient to introduce the functions

$$
g(s)=\frac{1}{\beta(s)} \quad \text { and } \quad G(s)=\left(g\left(s_{\mathrm{ref}}\right)-g(s)\right)\left(s-s_{\mathrm{ref}}\right)
$$

defined on $] 0, s_{\text {in }}[$, where $\beta$ is given in (3). Notice that function $g$ admits a unique minimum at $\hat{s}$ (by Lemma2.1) and satisfies $\lim _{s \rightarrow 0} g(s)=\lim _{s \rightarrow+\infty} g(s)=+\infty$. As we will see later, the function $G$ is proportional to the difference of the residence times between the one and two tanks configurations, for a given value of the substrate concentration in the lateral vessel.

The solution to the optimization problem 10 is given by the following proposition.

\section{Proposition 3.1 Define}

$$
\alpha=\max \left(0, s_{\text {ref }}-\frac{Q}{d}\left(s_{\text {in }}-s_{\text {ref }}\right)\right) .
$$

The solution of problem 10 satisfies:

(i) If $\hat{s} \leq \alpha$, then $V_{1}^{\mathrm{opt}}=0$ and $V_{2}^{\mathrm{opt}}=d g(\alpha)\left(s_{\mathrm{ref}}-\alpha\right)$.

(ii) If $\hat{s} \in] \alpha, s_{\mathrm{ref}}\left[\right.$, then $V_{1}^{\mathrm{opt}}=Q / \mu\left(s_{\mathrm{ref}}\right)+d g\left(s_{\mathrm{ref}}\right)\left(s_{2}^{\mathrm{opt}}-s_{\mathrm{ref}}\right)$ and $V_{2}^{\mathrm{opt}}=d g\left(s_{2}^{\mathrm{opt}}\right)\left(s_{\mathrm{ref}}-s_{2}^{\mathrm{opt}}\right)$, where

$$
s_{2}^{\mathrm{opt}}= \begin{cases}s_{G}, & \text { if } \alpha \in\left[0, s_{G}\right], \\ \alpha, & \text { if } \alpha \in] s_{G}, \hat{s}[\end{cases}
$$

$s_{G}$ being the unique minimum of the function $G$ on the interval $\left[\alpha, s_{\mathrm{ref}}\right]$. Moreover, $G^{\prime}\left(s_{2}^{\mathrm{opt}}\right)>0$ when $s_{2}^{\mathrm{opt}}=\alpha$.

(iii) If $\hat{s} \geq s_{\mathrm{ref}}$, then $V_{1}^{\mathrm{opt}}=Q / \mu\left(s_{\mathrm{ref}}\right)$ and $V_{2}^{\mathrm{opt}}=0$.

Proof. We replace the value of $s_{1}$ in system 21-22 by $s_{\text {ref }}$ :

$$
\left\{\begin{array}{l}
0=\frac{Q}{V_{1}}\left(s_{\text {in }}-s_{\text {ref }}\right)+\frac{d}{V_{1}}\left(s_{2}-s_{\text {ref }}\right)-\mu\left(s_{\text {ref }}\right)\left(s_{\text {in }}-s_{\text {ref }}\right), \\
0=\frac{d}{V_{2}}\left(s_{\text {ref }}-s_{2}\right)-\mu\left(s_{2}\right)\left(s_{\text {in }}-s_{2}\right) .
\end{array}\right.
$$


Considering function $g$, system 12 can be written as

$$
\left\{\begin{array}{l}
V_{1}=Q g\left(s_{\mathrm{ref}}\right)\left(s_{\mathrm{in}}-s_{\mathrm{ref}}\right)+d g\left(s_{\mathrm{ref}}\right)\left(s_{2}-s_{\mathrm{ref}}\right):=v_{1}\left(s_{2}\right), \\
V_{2}=d g\left(s_{2}\right)\left(s_{\mathrm{ref}}-s_{2}\right):=v_{2}\left(s_{2}\right) .
\end{array}\right.
$$

Thus, given model parameters $d, Q, s_{\text {in }}$ and $s_{\text {ref }}$, the volumes are completely characterized by variable $s_{2}$ and solving the optimization problem 10 is equivalent to look for solutions of the problem

$$
\min _{s_{2} \in \mathcal{S}_{2}} v_{1}\left(s_{2}\right)+v_{2}\left(s_{2}\right)
$$

where $\mathcal{S}_{2}$ is the set of admissible values of $s_{2}$. That is, the solution of problem 10 is given by $\left(v_{1}\left(s_{2}^{\text {opt }}\right), v_{2}\left(s_{2}^{\mathrm{opt}}\right)\right)$, where $s_{2}^{\text {opt }}$ is solution of problem (14). In order to determine the admissible set $\mathcal{S}_{2}$, we take into account that both values $V_{1}, V_{2}$ must be non-negative and proceed as follows:

(a) $v_{1}\left(s_{2}\right) \geq 0 \Leftrightarrow Q g\left(s_{\text {ref }}\right)\left(s_{\text {in }}-s_{\text {ref }}\right)+d g\left(s_{\text {ref }}\right)\left(s_{2}-s_{\text {ref }}\right) \geq 0 \Leftrightarrow s_{2} \geq s_{\text {ref }}-\frac{Q}{d}\left(s_{\text {in }}-s_{\text {ref }}\right)$.

(b) $v_{2}\left(s_{2}\right) \geq 0 \Leftrightarrow d g\left(s_{2}\right)\left(s_{\text {ref }}-s_{2}\right) \geq 0 \Leftrightarrow s_{2} \leq s_{\text {ref }}$.

Moreover, we have to impose variable $s_{2}$ to be non-negative, since it describes a (substrate) concentration. One concludes that $\mathcal{S}_{2}=\left[\alpha, s_{\text {ref }}\right]$.

For analytical purposes, we rewrite problem (14) as

$$
\min _{s_{2} \in\left[\alpha, s_{\mathrm{ref}}\right]} Q \underbrace{g\left(s_{\mathrm{ref}}\right)\left(s_{\mathrm{in}}-s_{\mathrm{ref}}\right)}_{A}+d G\left(s_{2}\right)
$$

The term $Q A$ corresponds to the optimal volume obtained with a single tank, and with a view to reduce this value, we aim to characterize solutions of problem 15 with values of the function $G$ being negative.

The cases considered in the proposition statement are treated separately.

(i) $\hat{s} \leq \alpha$ : Since function $g$ is increasing on the right of $\hat{s}$, then $g\left(s_{\text {ref }}\right) \geq g\left(s_{2}\right)$ for all $s_{2} \in\left[\alpha, s_{\text {ref }}\right]$. Consequently, function $G$ is negative on $\left[\alpha, s_{\text {ref }}\right]$ and is minimized for $s_{2}^{\text {opt }}=\alpha$.

(ii) $\hat{s} \in] \alpha, s_{\text {ref }}\left[\right.$ : In order to find $s_{2}^{\text {opt }}$ on $\left[\alpha, s_{\text {ref }}\right]$ such that $G\left(s_{2}^{o p t}\right)$ is minimum, we look for critical points of $G$, which satisfy

$$
g^{\prime}(s)=H(s):=\frac{g\left(s_{\mathrm{ref}}\right)-g(s)}{s-s_{\mathrm{ref}}} .
$$

By construction, function $g^{\prime}$ is increasing on $] 0, s_{\text {in }}\left[, g^{\prime}(\hat{s})=0\right.$ (since $g$ is strictly convex, being equal to $1 / \beta$ and $\beta$ strictly concave by Lemma 2.1], $g^{\prime}(\cdot)<0$ on $] 0, \hat{s}\left[\right.$ and $g^{\prime}(\cdot)>0$ on $] \hat{s}, s_{\text {in }}[$. Moreover, it is easy to see that the equation $H(s)=0$ has two solutions (and not more, as $g$ is strictly convex): $s_{\text {ref }}$ and $\bar{s}_{\text {ref }}:=\{s \in] 0, \hat{s}\left[: g\left(\bar{s}_{\text {ref }}\right)=\right.$ $\left.g\left(s_{\text {ref }}\right)\right\}$. In addition, it follows that $H(\cdot)>0$ on $\left[0, \bar{s}_{\text {ref }}[\right.$ and $H(\cdot)<0$ on $] \bar{s}_{\text {ref }}, s_{\text {ref }}[$. As a result, we can state 
that any critical point of function $G$ belongs to the interval $] \bar{s}_{\text {ref }}, \hat{s}[$.

We show that there exist a unique critical point $\left.s_{G} \in\right] \bar{s}_{\text {ref }}, \hat{s}[$ of $G$ by proving that function $H$ is decreasing on this interval:

$$
H^{\prime}(s)=\frac{-g^{\prime}(s)\left(s-s_{\mathrm{ref}}\right)-\left(g\left(s_{\mathrm{ref}}\right)-g(s)\right)}{\left(s-s_{\mathrm{ref}}\right)^{2}}=-\frac{g^{\prime}(s)+H(s)}{s-s_{\mathrm{ref}}}<0 .
$$

Since we look for the minimum value of function $G$ on the interval $\left[\alpha, s_{\mathrm{ref}}\right]$, one has that $s_{2}^{\text {opt }}$ depends on the value of $\alpha$. A direct conclusion is that $G^{\prime}\left(s_{2}^{\text {opt }}\right)=0$ when $s_{2}^{\text {opt }}=s_{G}$, while $G^{\prime}\left(s_{2}^{\text {opt }}\right)>0$ when $s_{2}^{\text {opt }}=\alpha$.

(iii) $s_{\text {ref }} \leq \hat{s}$ : One has that $s_{2} \leq s_{\text {ref }} \leq \hat{s}$ for all $s_{2} \in\left[\alpha, s_{\text {ref }}\right]$. Since function $g$ is decreasing on the left of $\hat{s}$, then $g\left(s_{\text {ref }}\right) \leq g\left(s_{2}\right)$. Consequently, function $G$ is non-negative on $\left[\alpha, s_{\text {ref }}\right]$ and the optimal value which makes it equal to zero is $s_{2}^{\text {opt }}=s_{\text {ref }}$.

Remark 3.1 From Proposition 3.1. one concludes that the particular configuration with $V_{1}=0$ (as the one depicted in Figure 33 is optimal if $\hat{s} \leq \alpha$ or $s_{G}<\alpha<\hat{s}<s_{\text {ref }}$.

\subsection{Characterization of the Best Value of the Parameter $d$}

Here, we consider that it is possible to play with the communication rate between the two patches, in addition to the distribution of the volumes, which leads us to consider that the parameter $d$ can be also optimized.

Given a nominal desired value $s_{\text {ref }}<s_{\text {in }}$ as output of the process, we look for solutions of the optimization problem

$$
\min _{\left(V_{1}, V_{2}, d\right) \in \mathbb{R}_{+}^{3}}\left\{V_{1}+V_{2} \text { such that } s_{1}=s_{\text {ref }} \text { at steady state }\right\},
$$

that we denote by $\left(V_{1}^{*}, V_{2}^{*}, d^{*}\right)$.

Proposition 3.2 The solution of problem (16) satisfies:

(i) If $\hat{s}<s_{\mathrm{ref}}$, then $V_{1}^{*}=0, V_{2}^{*}=Q\left(s_{\mathrm{in}}-s_{\mathrm{ref}}\right) g(\hat{s})$ and $d^{*}=Q \frac{s_{\mathrm{in}}-s_{\mathrm{ref}}}{s_{\mathrm{ref}}-\hat{s}}$.

(ii) If $\hat{s} \geq s_{\mathrm{ref}}$, then $V_{1}^{*}=Q / \mu\left(s_{\mathrm{ref}}\right), V_{2}^{*}=0$ and $d^{*}$ can take any value on the interval $[0,+\infty[$.

Proof. In order to solve problem (16), we rely on the optimization results obtained in Section 3.1. Thus, $\left(V_{1}^{*}, V_{2}^{*}, d^{*}\right)=$ $\left(V_{1}^{\mathrm{opt}}\left(d^{*}\right), V_{2}^{\mathrm{opt}}\left(d^{*}\right), d^{*}\right)$, where $d^{*}$ minimizes $V_{1}^{\mathrm{opt}}(d)+V_{2}^{\mathrm{opt}}(d)$ and $V_{1}^{\mathrm{opt}}, V_{2}^{\mathrm{opt}}$ are given by Proposition 3.1

(i) From Proposition 3.1, one easily deduces that the total volume $V^{\mathrm{opt}}(d)=V_{1}^{\mathrm{opt}}(d)+V_{2}^{\mathrm{opt}}(d)$ fulfills

$$
V^{\mathrm{opt}}(d)= \begin{cases}\frac{Q}{\mu\left(s_{\mathrm{ref}}\right)}+d G\left(s^{\mathrm{opt}}(d)\right), & \text { if } 0 \leq d<d^{*}(\text { case (ii) in Proposition 3.1), } \\ Q\left(s_{\mathrm{in}}-s_{\mathrm{ref}}\right) g\left(s_{\mathrm{ref}}-\frac{Q}{d}\left(s_{\mathrm{in}}-s_{\mathrm{ref}}\right)\right), & \text { if } d \geq d^{*}(\text { case (i) in Proposition 3.1), }\end{cases}
$$


where $s^{\text {opt }}$ must be now seen as a function of parameter $d$.

We analyze the monotonicity of function $V^{\mathrm{opt}}$.

(a) When $0 \leq d<d^{*}$, one has that

$$
\frac{\partial V^{\mathrm{opt}}}{\partial d}=G\left(s^{\mathrm{opt}}(d)\right)-\left.d \frac{\partial G}{\partial s}\right|_{s=s^{\mathrm{opt}}(d)} \frac{\partial s^{\mathrm{opt}}(d)}{\partial d}
$$

From Proposition 3.1, it follows that having $G\left(s^{\mathrm{opt}}(d)\right)<0$ and $s^{\mathrm{opt}}(d)$ corresponds either to $s^{G}\left(\right.$ with $\left.G^{\prime}\left(s^{G}\right)=0\right)$ or to $\alpha$ (with $G^{\prime}(\alpha)>0$ ). In both cases one has $\frac{\partial V^{\mathrm{opt}}}{\partial d}<0$, that is, $V^{\text {opt }}$ is decreasing on $\left[0, d^{*}[\right.$.

(b) When $d \geq d^{*}$, one has that

$$
\frac{\partial V^{\text {opt }}}{\partial d}=\frac{Q^{2}}{2 d^{2}}\left(s_{\text {in }}-s_{\text {ref }}\right)^{2} g^{\prime}\left(s_{\text {ref }}-\frac{Q}{d}\left(s_{\text {in }}-s_{\text {ref }}\right)\right) .
$$

By definition, $\hat{s}$ is the only value satisfying $g^{\prime}(\hat{s})=0$ and so $d^{*}$ is the only critical point of function $V^{\text {opt }}$ It remains to prove that $d^{*}$ is a minimum of function $V^{\mathrm{opt}}$. But one has

$$
\frac{\partial^{2} V^{\mathrm{opt}}}{\partial d^{2}}\left(d^{*}\right)=\frac{Q^{3}}{4\left(d^{*}\right)^{4}}\left(s_{\mathrm{in}}-s_{\mathrm{ref}}\right)^{3} g^{\prime \prime}(\hat{s}),
$$

which is positive as $g$ is strictly convex. Therefore $V^{\text {opt }}$ is increasing on $\left[d^{*}, \infty[\right.$.

From these two points we conclude that the optimal value of $d$ is $d^{*}$.

(ii) This is a direct consequence of the statement (iii) in Proposition 3.1, since in this case the optimal volumes, solution of problem 10 , do not depend on parameter $d$.

\subsection{Numerical Illustration}

One may wonder how much could be gained (in terms of residence time or total volume) by using the proposed structure. Nevertheless, it is difficult to quantify the overall profit since the optimal design depends on parameters $s_{\text {in }}$, $s_{\text {ref }}, Q, \mu(\cdot)$ and $d$ (when it is not fixed beforehand). As an illustrative example, we compare the total optimal volumes $V^{\mathrm{opt}}(0), V^{\mathrm{opt}}(Q)$ and $V^{\mathrm{opt}}\left(d^{*}\right)$ obtained by solving problem 10 for the Monod Function (2) with the following parameters

\begin{tabular}{|c|c|c|c|}
\hline$\mu_{\max }$ & $K$ & $Q$ & $s_{\text {in }}$ \\
\hline \hline 1 & 0.5 & 1 & 10 \\
\hline
\end{tabular}

and different values of the reference concentration $s_{\text {ref }}$. In this case one obtains $\hat{s} \approx 1.79$. The value of the diffusion coefficient $d$ has been taken equal to $0, Q$ and $d^{*}$, respectively. The case $d=Q$ responds to the "simple gradostat" for which all the communication rates are identical $7,3,33,34$. For the optimal case $d=d^{\star}, d^{\star}$ depends on $s_{\text {ref }}$. 
Figure 4 (a) compares the three values of diffusion coefficient while the associated optimal volumes are depicted in Figure 4 (b). Notice that $V^{\mathrm{opt}}(0)$ corresponds to the volume of the single-tank chemostat.

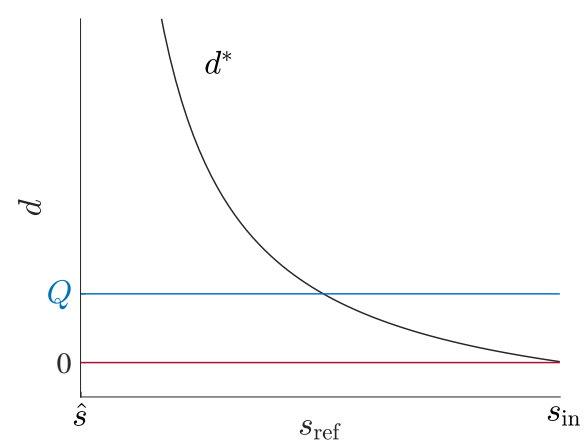

(a) Considered diffusion parameters (seen as functions of $\left.s_{\text {ref }}\right)$.

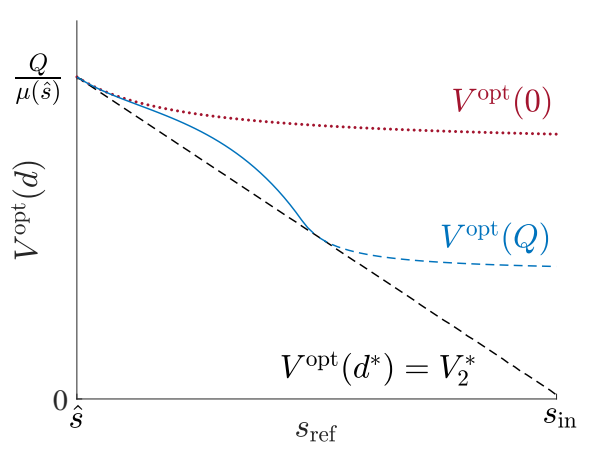

(b) Optimal volumes associated to the diffusion parameters in (a).

Fig. 4 Comparison of volumes $V^{\mathrm{opt}}(0), V^{\mathrm{opt}}(Q)$ and $V^{\mathrm{opt}}\left(d^{*}\right)$ necessary to obtain $s_{\text {ref }}$ at output of the main vessel. The solid, dashed and dotted lines in (b) represent, respectively, the values of $s_{\text {ref }}$ for which the optimal design is composed of two tanks (of volumes $V_{1}^{\text {opt }}$ and $V_{2}^{\mathrm{opt}}$ ), a single tank of volume $V_{2}^{\mathrm{opt}}$ or a single tank of volume $V_{1}^{\mathrm{opt}}$.

From Figure 4 (b) we remark that, when $d=Q$, there exists a certain value of parameter $s_{\text {ref }}$ in which the optimal

design transits from having two to one tank. Proposition 3.2 infers that this transition occurs when $s_{\text {ref }}=\frac{s_{\text {in }}+\hat{s}}{2}$ (in this case, one has $s_{\text {ref }} \approx 5.9$ ). One can observe that, for this particular value of $s_{\text {ref }}$, the single-tank volume is reduced approximately to its half and, in general, the volume reduction becomes more significant as the value $s_{\text {ref }}$ increases. In those cases the gains are quite significant.

\section{Discussion and Interpretation of the Results}

This section is devoted to the analysis of the impact of the lateral diffusion from both ecological and economic points of view.

\subsection{In Terms of Ecological Impact}

In Section 2, we have investigated the conversion yield of the proposed structured chemostat and compared it with the one of a single-tank chemostat. Our main result, presented in Proposition 2.3. can be interpreted depending on the global removal rate $D=Q / V$ and a threshold $\hat{s}$ (that is defined as the maximizer of the function $\beta$ defined in (3)) as follows: 
1. If $D>\mu\left(s_{\text {in }}\right)$, a spatial distribution of the total volume $V$ could avoid the extinction of the micro-organisms while it happens when the volume $V$ is perfectly mixed. Therefore, the lateral-diffusive compartment plays the role of a "refuge" for the micro-organisms under large removal rates.

2. If $D \in\left[\mu(\hat{s}), \mu\left(s_{\text {in }}\right)\right]$, a spatial distribution of the total volume $V$ increases systematically the output substrate concentration with respect to that obtained if the volume $V$ would be perfectly mixed. Spatialization is thus a penalizing factor for the conversion yield.

3. If $D<\mu(\hat{s})$, a spatial distribution of the total volume $V$ could reduce the output substrate concentration obtained when the volume $V$ is perfectly mixed (and therefore improves the conversion yield), but this is not systematic. This means that for small removal rates $D$ (as often met, for instance, in soil ecosystems) one cannot know if a perfectly mixed model under- or over-estimates the expected output level of conversion.

We have also analyzed the influence of the diffusion parameter $d$ on the conversion yield in cases 1 and 3 and shown the existence of a most efficient value $d^{\star}$. Finally, this study reveals that a lateral-diffusive compartment is beneficial only for contrasted situations (i.e., large or small removal rates), which does not appear to be an intuitive property.

\subsection{In Terms of Economic Benefits}

In Section 3, we studied optimal choices of the main design parameters (tanks volumes and diffusion rate) that minimize the residence time (or equivalently the total required volume) for a given conversion yield. Our main result, presented in Propositions 3.1 and 3.2 states that, when the desired substrate output concentration is above certain threshold (more precisely, when $s_{\text {ref }}>\hat{s}$ ), the volume of a single-tank chemostat can be reduced by using the structure with lateral diffusion. This result complements the work in $29,53,55,57,58$, where the authors propose a methodology to diminish the volume of a single-tank chemostat when $s_{\text {ref }} \leq \hat{s}$, by using either $N$ CSTR ("Continuous Stirred Tank Reactor") in series or a CSTR connected in series to a PFR ("Plug Flow Reactor"). We distinguish between the following cases:

a. The diffusion coefficient between the two tanks is imposed by the hydrodynamical conditions. Depending on model parameters $s_{\text {in }}, s_{\text {ref }}, Q, d$ and $\mu(\cdot)$, the optimal structure may be composed of two tanks (of non null volumes $V_{1}^{\text {opt }}$ and $V_{2}^{\text {opt }}$ ) or a a single lateral tank (of volume $V_{2}^{\text {opt }}$ ) connected by diffusion to the main stream.

b. The communication rate of substrate and biomass between the two tanks can be adjusted, typically by means of membrane or pair of pumps in each direction. The diffusion coefficient can be then optimized as well. The optimal structure is necessarily a single lateral tank (of volume $V_{2}^{\mathrm{opt}}$ ) connected by diffusion (with optimal diffusion rate $\left.d^{*}=Q \frac{s_{\text {in }}-s_{\text {ref }}}{s_{\text {ref }}-\hat{s}}\right)$ to the main stream.

So an important message of this study is that the particular structure of a single tank connected by diffusion to a pipe that conducts the input stream, as depicted on Figure 3, can be an efficient configuration, better than a single 
tank directly under the main stream. To our knowledge, this result is new in the literature.

The mathematical analysis has also revealed that the function $g$, i.e. the inverse of the function $\beta$ defined in (3), is playing an important role in determining if the best configuration is composed of one or two tanks (more precisely the relative position of the output reference value $s_{\text {ref }}$ with respect to the minimizer $\hat{s}$ of $g$ ). It has to be underlined that this is exactly the same function than the one used for the optimal design of tanks in series (for which a discussion about the relative position of $s_{\text {ref }}$ with respect to $\hat{s}$ informs if one or two tanks in series is the best, see, e.g., 53, 54]), but with two main differences here:

1. Due to the particular considered structure, there is a trichotomy (one single mixed tank, two tanks, or one single lateral tank) instead of the dichotomy (one or more tanks) found for the problem with tanks in series. This trichotomy is discussed below with the help of the additional parameter $\alpha=\max \left(0, s_{\text {ref }}-\frac{Q}{V}\left(s_{\text {in }}-s_{\text {ref }}\right)\right)$.

2. For small values of $s_{\text {ref }}$ (compared to $\hat{s}$ ), a lateral-diffusion compartment does not bring any improvement compared to a single perfectly mixed tank, while this is the opposite for tanks in series (i.e. several tanks are better than a single one when $\left.s_{\text {ref }}<\hat{s}\right)$.

These points can be grasped by the following graphical interpretation. Consider the total volume $V$ required to obtain the output concentration $s_{\text {ref }}$ at steady state. In our case, it can be written in terms of the function $g$ as follows

$$
V=Q \underbrace{g\left(s_{\mathrm{ref}}\right)\left(s_{\mathrm{in}}-s_{\mathrm{ref}}\right)}_{A}+d \underbrace{\left(g\left(s_{\mathrm{ref}}\right)-g\left(s_{2}^{\star}\right)\right)\left(s_{2}^{\star}-s_{\mathrm{ref}}\right)}_{B}
$$

where $s_{2}^{\star}$ is the steady state in the second compartment. One can notice that the number $A$ is proportional to the volume necessary for a single chemostat to have $s_{\text {ref }}$ as substrate concentration at steady state. Therefore, a configuration with a lateral-diffusive compartment would require a smaller volume than that of the single chemostat exactly when the number $B$ is negative. Figure 5 illustrates that this is possible only when $s_{\text {ref }}$ is above the minimizer $\hat{s}$ of the function $g$. Furthermore, the quantity $B$ is equal to $G\left(s_{2}^{\star}\right)$, where the function $G$ defined in (11) admits a unique minimum at $s_{G} \in\left[0, s_{\text {ref }}\right]$.

Proposition 3.1 states that, when $s_{\text {ref }}>\hat{s}$, the optimal value of $s_{2}^{\star}$ is $s_{G}$ when $\alpha \leq s_{G}$ and $\alpha$ in other case, the later scenario corresponding to the particular configuration with $V_{1}=0$. A graphical interpretation of the optimized structures obtained when parameter $d$ is fixed is given in Figure 6. These pictures read as follows. In (a) and (b), the main tank is not void and $Q A$ represents the volume necessary for a single chemostat when operating at the desired steady state $s_{\text {ref }}$ (without diffusion). In case (a), it is optimal to have a single tank, whose volume is thus $Q A$. In case (b), $d B$ represents the optimal volume of the lateral tank and $d C$ the amount of volume that is gained on the main tank compared to the single tank configuration. Thus, one has $V_{1}=Q A-d C$ and $V_{2}=d B$ as optimal configuration. Finally, on (c) the limiting of $V_{1}=0$ highlighted in Remark 2.2 is optimal with $V_{2}=d B$, accordingly to Proposition 


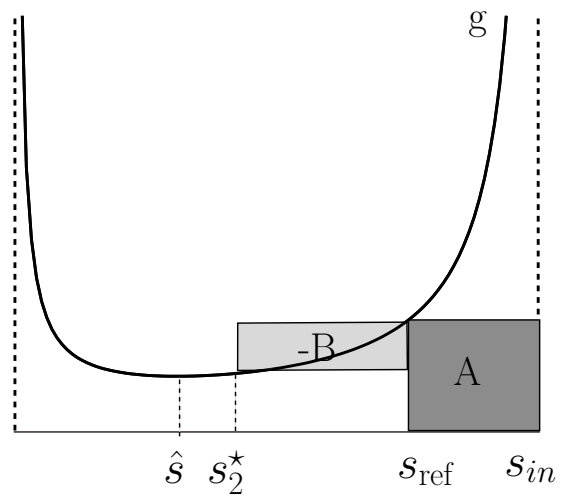

(a) $s_{\text {ref }}>\hat{s}$ ( $B$ is negative)

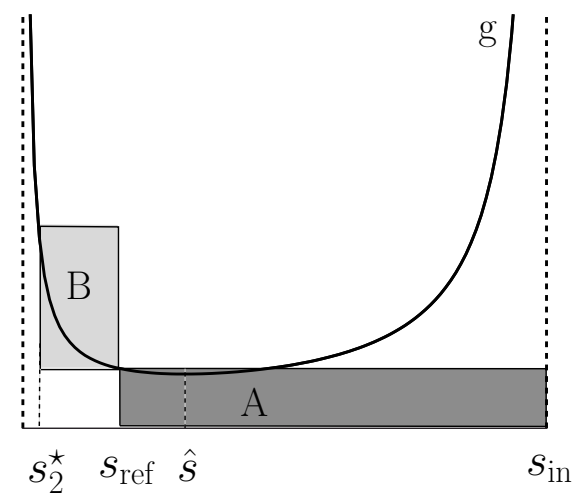

(b) $s_{\text {ref }}<\hat{s}$ ( $B$ is positive)

Fig. 5 Graphical representation of quantities $A$ and $|B|$ in 17.

3.2 Notice that all the quantities $A, B, C$ can be determined graphically, once the numbers $s_{G}$ and $\alpha$ are computed. Only $\alpha$ depends on the parameter $d$. When the diffusion rate can be chosen, the optimized configurations are cases (a) or (c), depending of the position of $s_{\text {ref }}$ relatively to $\hat{s}$, but never case (b).

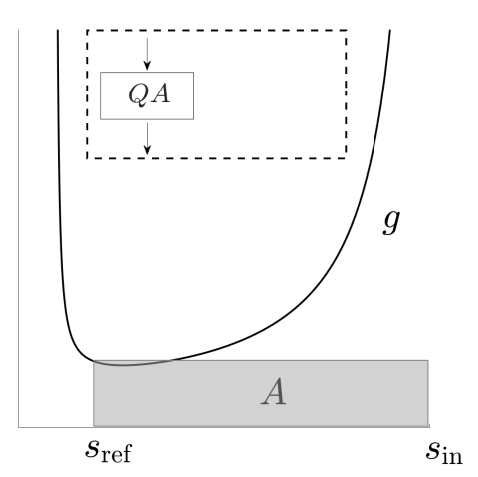

(a) $s_{\text {ref }}<\hat{s}$

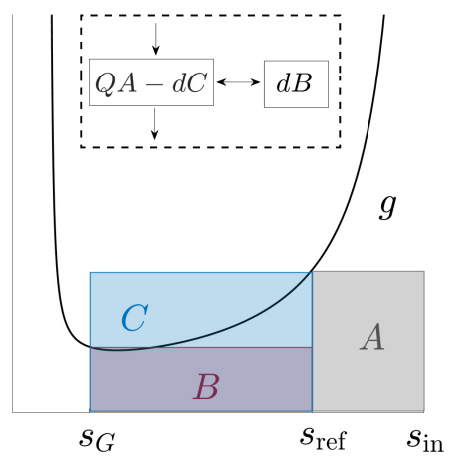

(b) $\alpha \leq s_{G}<\hat{s}<s_{\text {ref }}$

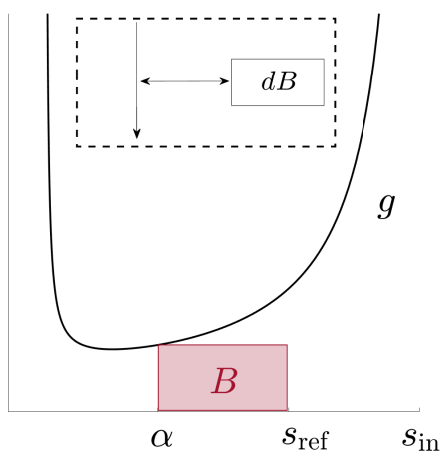

(c) $\hat{s} \leq \alpha$ or $s_{G}<\alpha<\hat{s}<s_{\text {ref }}$

Fig. 6 Graphical representation of the optimized configurations when parameter $d$ is fixed.

Finally, let us recall from the theory of optimal design of chemostats in series that the first tank (when it is optimal to have more than one tank) has systematically a substrate concentration $s_{1}^{\star}$ above $\hat{s}$ at steady state (see, e.g., [53 54]). Thus, for a economic view point, we can state that a lateral-diffusive compartment for the first tank of an optimal series of chemostats could systematically improve the performance of the overall process. 


\section{Conclusions}

In this work, we have identified situations for which the chemostat pattern with a compartment connected by "lateral diffusion" is beneficial for ecological or economic considerations, and characterized the optimal configurations. We have also proposed graphical interpretations of the results.

The analysis has first revealed two thresholds on the input nutrient concentration which allow to distinguish three kind of situations for guaranteeing the existence of a positive equilibrium depending on the diffusion rate $d$ : I. $d$ has to be positive but below a maximal value $\bar{d}$, II. $d$ has simply to be positive, III. $d$ can take any non-negative value. When the positive equilibrium exists, we have proved that it is necessary asymptotically stable (although not necessarily hyperbolic).

We have also studied the impact of the diffusion rate $d$ on the conversion yield and concluded that there exists an optimal value of $d$ that maximizes the conversion yield (which is then necessarily better than for a single tank) for either small or large values of the removal rate, which did not appear to be an intuitive result to us. This property implies that in natural habitats (such as in soil ecosystems) the type of feeding (by convection or diffusion) from a given flow rate could have a significant impact on the effective conversion yield.

In terms of residence time (or equivalently total volume) required for achieving a given conversion yield, we have provided conditions that discriminate which configurations between one or two tanks are the best. Our conclusion is that a lateral compartment is beneficial compared to a single chemostat when the conversion yield is not too important (i.e. when the output concentration is not too small compared to the input one). Surprisingly, we have also found that the limiting case of a single tank purely connected by diffusion to the advective stream (as depicted on Figure 3, and not crossed by the stream as in the classical chemostat, can provide the minimal volume. For economic benefits, we can state that a lateral diffusive compartment for the first tank of an optimal series of chemostat could systematically improve the performance of the overall process. Therefore, the analysis of combinations of series and lateral diffusive compartments (which is out of the scope of the present study) would most probably exhibit other non-intuitive configurations that have not yet been considered in the literature. Consideration of biomass attachment or biofilms (leading to different diffusion coefficients for the substrate and the micro-organisms) should be also relevant to study. 


\section{Appendix: Proofs of the Primary Results of Section 2 .}

Proof of Lemma 2.2

Define $z_{i}=s_{\text {in }}-s_{i}-x_{i}$ for each tank $i=1,2$ and consider the dynamics 11 in $(\mathbf{z}, \mathbf{s})$ coordinates:

$$
\left\{\begin{array}{l}
\dot{z}_{1}=-\frac{Q}{V_{1}} z_{1}-\frac{d}{V_{1}}\left(z_{1}-z_{2}\right), \\
\dot{s}_{1}=-\mu\left(s_{1}\right)\left(s_{\text {in }}-s_{1}-z_{1}\right)+\frac{Q}{V_{1}}\left(s_{\text {in }}-s_{1}\right)+\frac{d}{V_{1}}\left(s_{2}-s_{1}\right), \\
\dot{z}_{2}=-\frac{Q}{V_{2}}\left(z_{2}-z_{1}\right) \\
\dot{s}_{2}=-\mu\left(s_{2}\right)\left(s_{\text {in }}-s_{2}-z_{2}\right)+\frac{d}{V_{2}}\left(s_{1}-s_{2}\right) .
\end{array}\right.
$$

This system has a cascade structure with a first independent sub-system linear in $\mathbf{z}$

$$
\dot{\mathbf{z}}=\underbrace{\left[\begin{array}{cc}
-\frac{Q+d}{V_{1}} & \frac{d}{V_{1}} \\
\frac{d}{V_{2}} & -\frac{d}{V_{2}}
\end{array}\right]}_{\mathbf{M}} \mathbf{z},
$$

where one has

$$
\operatorname{tr}(\mathbf{M})=-\frac{Q+d}{V_{1}}-\frac{d}{V_{2}}<0 \text { and } \operatorname{det}(\mathbf{M})=\frac{Q d}{V_{1} V_{2}}>0 .
$$

Therefore the matrix $\mathbf{M}$ is Hurwitz and any solution $\mathbf{z}$ of 19 converges exponentially to $\mathbf{0}$. Then, the solution $\mathbf{s}$ can be written as the solution of the non-autonomous dynamics

$$
\dot{\mathbf{s}}=\mathbf{F}(t, \mathbf{s})=\left[\begin{array}{c}
\left(\frac{Q}{V_{1}}-\mu\left(s_{1}\right)\right)\left(s_{\text {in }}-s_{1}\right)+\frac{d}{V_{1}}\left(s_{2}-s_{1}\right)+\mu\left(s_{1}\right) z_{1}(t) \\
-\mu\left(s_{2}\right)\left(s_{\text {in }}-s_{2}\right)+\frac{d}{V_{2}}\left(s_{1}-s_{2}\right)+\mu\left(s_{2}\right) z_{2}(t)
\end{array}\right] .
$$

Notice that, for any $(t, \mathbf{s})$, one has

$$
\frac{\partial F_{1}(t, \mathbf{s})}{\partial s_{2}}=\frac{d}{V_{1}}>0 \quad \text { and } \quad \frac{\partial F_{2}(t, \mathbf{s})}{\partial s_{1}}=\frac{d}{V_{2}}>0,
$$

and so the dynamics 20 is cooperative (see, e.g., 56]).

Define $\check{F}_{1}(t, \mathbf{s}):=-\frac{Q}{V_{1}} s_{1}-\mu\left(s_{1}\right)\left(s_{\text {in }}-s_{1}\right)+\frac{d}{V_{1}}\left(s_{2}-s_{1}\right)+\mu\left(s_{1}\right) z_{1}(t)$, for which it follows that $F_{1}(t, \mathbf{s})>\check{F}_{1}(t, \mathbf{s})$ for any $(t, \mathbf{s})$. Proposition 2.1 in [56] allows to state that any solution of 20] with $s_{i}(0) \geq 0(i=1,2)$ satisfies $s_{i}(t) \geq \check{s}_{i}(t)(i=1,2)$ for any $t>0$, where $\check{\mathbf{s}}$ is solution of the dynamics

$$
\dot{\check{\mathbf{s}}}=\check{\mathbf{F}}(t, \check{\mathbf{s}})=\left[\begin{array}{l}
\check{F}_{1}(t, \check{\mathbf{s}}) \\
F_{2}(t, \check{\mathbf{s}})
\end{array}\right], \quad \check{\mathbf{s}}(0)=\mathbf{0}
$$

As one has $\check{\mathbf{F}}(t, \mathbf{0})=\mathbf{0}$ for any $t$, the solution $\check{\mathbf{s}}$ is identically null and one obtains that $s_{i}(t)(i=1,2)$ stays non-negative for any positive $t$. Similarly, $\mathbf{x}$ can be written as a solution of a non-autonomous cooperative dynamics

$$
\dot{\mathbf{x}}=\mathbf{L}(t, \mathbf{x})=\left[\begin{array}{c}
\left(\mu\left(s_{1}(t)\right)-\frac{Q+d}{V_{1}}\right) x_{1}+\frac{d}{V_{1}} x_{2} \\
\frac{d}{V_{2}} x_{1}+\left(\mu\left(s_{2}(t)\right)-\frac{d}{V_{2}}\right) x_{2}
\end{array}\right]
$$

with $\mathbf{L}(t, \mathbf{0})=\mathbf{0}$, which allows to conclude that $x_{i}(t)(i=1,2)$ stays non-negative for any positive $t$.

Finally, the convergence of $\mathbf{z}$ to $\mathbf{0}$ provides the boundedness of the solutions $\mathbf{s}(t), \mathbf{x}(t)$.

Proof of Proposition 2.1.

From the two last equations of (1), one has $s_{1}+x_{1}=s_{2}+x_{2}$ at steady-state, and from the two first ones $s_{1}+x_{1}=s_{\text {in }}$. The values $s_{1}, s_{2}$ at steady state are then solutions of the system of two equations

$$
\begin{aligned}
& 0=\left(\frac{Q}{V_{1}}-\mu\left(s_{1}\right)\right)\left(s_{\text {in }}-s_{1}\right)+\frac{d}{V_{1}}\left(s_{2}-s_{1}\right) \\
& 0=-\mu\left(s_{2}\right)\left(s_{\text {in }}-s_{2}\right)+\frac{d}{V_{2}}\left(s_{1}-s_{2}\right)
\end{aligned}
$$

and $x_{1}, x_{2}$ at steady state are uniquely defined from each solution $\left(s_{1}, s_{2}\right)$ of 21-22. Clearly, $\left(s_{\text {in }}, s_{\text {in }}\right)$ is a solution of 21)-22). We look for (positive) solutions different to $\left(s_{\text {in }}, s_{\text {in }}\right)$. Posit

$$
\lambda_{1}\left(s_{\text {in }}\right):=\max \left\{s_{1} \in\left[0, s_{\text {in }}\right]: \mu\left(s_{1}\right) \leq \frac{Q}{V_{1}}\right\}
$$


From equations 21]-22, a solution different to $\left(s_{\text {in }}, s_{\text {in }}\right)$ has to satisfy $s_{1}>s_{2}>0$ and then from equation 21, one has also $s_{1}<\lambda_{1}\left(s_{\text {in }}\right)$. Define then the functions:

$$
\begin{aligned}
& \phi_{1}\left(s_{1}\right):=s_{1}-\frac{Q-V_{1} \mu\left(s_{1}\right)}{d}\left(s_{\text {in }}-s_{1}\right)=s_{1}-\frac{Q}{d}\left(s_{\text {in }}-s_{1}\right)+\frac{V_{1}}{d} \beta\left(s_{1}\right), \\
& \phi_{2}\left(s_{2}\right):=s_{2}+\frac{V_{2} \mu\left(s_{2}\right)}{d}\left(s_{\text {in }}-s_{2}\right)=s_{2}+\frac{V_{2}}{d} \beta\left(s_{2}\right) .
\end{aligned}
$$

so that any solution of 21)- 22 fulfills $s_{2}=\phi_{1}\left(s_{1}\right)$ and $s_{1}=\phi_{2}\left(s_{2}\right)$. One has

$$
\phi_{1}^{\prime}\left(s_{1}\right)=1+\frac{V_{1}}{d} \mu^{\prime}\left(s_{1}\right)\left(s_{\text {in }}-s_{1}\right)+\frac{Q-V_{1} \mu\left(s_{1}\right)}{d} .
$$

Therefore, $\phi_{1}$ is increasing on $\left[0, \lambda_{1}\left(s_{\text {in }}\right)\right]$, with $\phi_{1}(0)=-(Q / d) s_{\text {in }}<0$ and $\phi_{1}\left(\lambda_{1}\left(s_{\text {in }}\right)\right)=\lambda_{1}\left(s_{\text {in }}\right)>0$. Thus, $\phi_{1}$ is invertible on $\left[-(Q / d) s_{\text {in }}, \lambda_{1}\left(s_{\text {in }}\right)\right]$ with

$$
\left.\phi_{1}^{-1}(0) \in\right] 0, \lambda_{1}\left(s_{\text {in }}\right)[\text {. }
$$

From Lemma 2.1 it follows that $\phi_{1}$ and $\phi_{2}$ are strictly concave functions on $\left[0, s_{\text {in }}\right]$. Consider then the function

$$
\gamma\left(s_{2}\right)=\phi_{2}\left(s_{2}\right)-\phi_{1}^{-1}\left(s_{2}\right) \quad s_{2} \in\left[0, s_{\text {in }}\right],
$$

which is also strictly concave on $\left[0, s_{\mathrm{in}}\right]$. Then, a solution $\left(s_{1}, s_{2}\right)$ can be written as a solution of

$$
\gamma\left(s_{2}\right)=0, \quad s_{1}=\phi_{2}\left(s_{2}\right) \quad \text { with } s_{2} \in\left[0, \lambda_{1}\left(s_{\text {in }}\right)\right] .
$$

Notice that one has $\gamma\left(s_{\text {in }}\right)=0$, and as $\gamma$ is strictly concave, it cannot have more than two zeros. Therefore there is at most one solution $\left(s_{1}, s_{2}\right)$ different to $\left(s_{\mathrm{in}}, s_{\mathrm{in}}\right)$. Furthermore, one has $\gamma(0)=-\phi_{1}^{-1}(0)<0$. Now, distinguish two different cases:

- When $\lambda_{1}\left(s_{\text {in }}\right)<s_{\text {in }}$ (or equivalently $\left.\mu\left(s_{\text {in }}\right)>Q / V_{1}\right)$, one has

$$
\gamma\left(\lambda_{1}\left(s_{\text {in }}\right)\right)=\frac{Q V_{2}}{d V_{1}}\left(s_{\text {in }}-\lambda_{1}\left(s_{\text {in }}\right)\right)>0 .
$$

By using the Mean Value Theorem, one concludes that there exists $s_{2} \in\left(0, \lambda_{1}\left(s_{\text {in }}\right)\right)$ such that $\gamma\left(s_{2}\right)=0$.

- When $\lambda_{1}\left(s_{\text {in }}\right)=s_{\text {in }}$ (that is when $\left.\mu\left(s_{\text {in }}\right) \leq Q / V_{1}\right)$, the function $\gamma$ takes positive values on the interval $\left[0, s_{\text {in }}\right]$ if and only if $\gamma^{\prime}\left(s_{\text {in }}\right)<0$ ( $\gamma$ being strictly concave on $\left.\left[0, s_{\text {in }}\right]\right)$, or equivalently when the condition

$$
\phi_{2}^{\prime}\left(s_{\mathrm{in}}\right)<\frac{1}{\phi_{1}^{\prime}\left(s_{\mathrm{in}}\right)}
$$

is fulfilled. Notice that one has $\phi_{1}^{\prime}\left(s_{\text {in }}\right)>0$ because $\lambda_{1}\left(s_{\text {in }}\right)=s_{\text {in }}$. So the condition can be also written as $\phi_{1}^{\prime}\left(s_{\text {in }}\right) \phi_{2}^{\prime}\left(s_{\text {in }}\right)<1$. From the expressions of $\phi_{1}$ and $\phi_{2}$, one can write this condition as

$$
\frac{\left(d+Q-V_{1} \mu\left(s_{\text {in }}\right)\right)\left(d-V_{2} \mu\left(s_{\text {in }}\right)\right)}{d^{2}}<1
$$

and check that this exactly amounts to require $s_{\text {in }}$ to satisfy $P\left(\mu\left(s_{\text {in }}\right)\right)<0$.

We conclude that there exists a positive steady state if and only if $\mu\left(s_{\text {in }}\right)>Q / V_{1}$ or $P\left(\mu\left(s_{\text {in }}\right)\right)<0$ and that this steady state (when it exists) is unique.

Let us study now the stability of the steady-states. Due to the cascade structure of the dynamics 1 that is made explicit in the proof of Lemma 2.2. the Jacobian matrix in the $(\mathbf{z}, \mathbf{s})$ coordinates depends only on $\mathbf{s}$ and is equal to

$$
\mathbf{J}(\mathbf{s})=\left[\begin{array}{cc}
\mathbf{M} & \mathbf{0} \\
\mathbf{N}(\mathbf{s}) & \mathbf{J}_{a}(\mathbf{s})
\end{array}\right] \quad \text { with } \quad \mathbf{J}_{a}(\mathbf{s})=\left[\begin{array}{cc}
-\frac{d}{V_{1}} \phi_{1}^{\prime}\left(s_{1}\right) & \frac{d}{V_{1}} \\
\frac{d}{V_{2}} & -\frac{d}{V_{2}} \phi_{2}^{\prime}\left(s_{2}\right)
\end{array}\right], \mathbf{N}(\mathbf{s})=\left[\begin{array}{cc}
\mu\left(s_{1}\right) & 0 \\
0 & \mu\left(s_{2}\right)
\end{array}\right]
$$

where the matrix $\mathbf{M}$ defined in 19 is Hurwitz. Accordingly to Proposition 2.1 the equilibrium $\mathbf{E}^{\star} \neq \mathbf{E}^{0}$ exists when $P\left(\mu\left(s_{\text {in }}\right)\right)>0$ or $\mu\left(s_{\text {in }}\right)>Q / V_{1}$

- When $P\left(\mu\left(s_{\text {in }}\right)\right)>0$, one has $\phi_{1}^{\prime}\left(s_{\text {in }}\right) \phi_{2}^{\prime}\left(s_{\text {in }}\right)<1$ or equivalently $\operatorname{det}\left(\mathbf{J}_{a}\left(\mathbf{s}^{0}\right)\right)<0$. Then $\mathbf{E}^{0}$ is a saddle point (with a stable manifold of dimension one).

- When $\mu\left(s_{\text {in }}\right)>Q / V_{1}$, notice that the equilibrium $\mathbf{E}^{0}$ is not necessarily hyperbolic (as one can have $P\left(\mu\left(s_{\text {in }}\right)\right)=0$ which implies then $\left.\operatorname{det}\left(\mathbf{J}_{a}\left(\mathbf{s}^{0}\right)\right)=0\right)$ and we cannot conclude its stability properties directly.

As already mentioned in Lemma 2.2 the dynamics is cooperative in the $(\mathbf{z}, \mathbf{s})$ coordinates. Moreover it is irreducible when $\mu\left(s_{1}\right)$ or $\mu\left(s_{2}\right)$ is non null. But one has

$$
s_{i}=0 \Rightarrow \dot{s}_{i} \geq \frac{Q}{V_{i}} s_{i n}>0 \quad(i=1,2)
$$


The domain $\mathcal{D}=\mathbb{R}^{2} \times\left(\mathbb{R}_{+} \backslash\{0\}\right)^{2}$ is thus invariant and one can consider without loss of generality initial conditions in $\mathcal{D}$. Then, the dynamics is strongly monotone on $\mathcal{D}$. As any forward orbit of $[18$ in $\mathcal{D}$ is bounded (see Lemma 2.2 , we can use the property of strongly monotone systems (see for instance Theorem C.8 in 7) to conclude that for any initial condition of $(1)$ in $\mathbb{R}_{+}^{4}$, except on a set of null measure, the trajectory solution converges asymptotically to an equilibrium. Finally, when the equilibrium $\mathbf{E}^{\star}$ exists, the analysis conducted in the proof of Proposition 2.1 allows us to deduce the inequalities $\phi_{1}^{\prime}\left(s_{1}^{\star}\right)>0$ and $\gamma^{\prime}\left(s_{2}^{\star}\right)>0$, which in turn imply $\phi_{1}^{\prime}\left(s_{1}^{\star}\right) \phi_{2}^{\prime}\left(s_{2}^{\star}\right)>1$, and so $\phi_{2}^{\prime}\left(s_{2}^{\star}\right)>0$. Then, one has $\operatorname{tr}\left(\mathbf{J}_{a}\left(\mathbf{s}^{\star}\right)\right)<0$ and $\operatorname{det}\left(\mathbf{J}_{a}\left(\mathbf{s}^{\star}\right)\right)>0$ i.e. $\mathbf{J}\left(\mathbf{s}^{\star}\right)$ is Hurwitz, which proves that the attractive equilibrium $\mathbf{E}^{\star}$ is also locally exponentially stable.

Acknowledgements The authors thank the French LabEx NUMEV (project ANR-10-LABX-20), incorporated into the I-Site MUSE, for the postdoctoral grant of the First Author at MISTEA lab, Montpellier, France.

\section{References}

1. Monod, J.: La technique de la culture continue: théorie et applications. Ann. Inst. Pasteur 79, 390-410 (1950).

2. Novick, A., Szilard, L.: Description of the chemostat. Science 112, 715-716 (1950).

3. Hoskisson, P.A., Hobbs, G.: Continuous culture - making a comeback? Microbiology 151, 3153-3159 (2005). .

4. Harmand, J., Rapaport, A., Dochain, D., Lobry, C.: Microbial ecology and bioprocess control: Opportunities and challenges. J. Process Contr. 18(9), 865-875 (2008).

5. Wade, M., Harmand, J., Benyahia, B., Bouchez, T., Chaillou, S., Cloez, B., Godon, J.J., Lobry, C., Moussa Boubjemaa, B., Rapaport, A., Sari, T., Arditi, R.: Perspectives in Mathematical Modelling for Microbial Ecology. Ecol. Modell. 321, 64-74 (2016). .

6. Hsu, S.B., Hubbell, S., Waltman, P.: A mathematical theory for single-nutrient competition in continuous cultures of microorganisms. SIAM J. Appl. Math. 32, 366-383 (1977).

7. Smith, H., Waltman, P.: The theory of chemostat, dynamics of microbial competition. Cambridge Studies in Mathematical Biology, Cambridge University Press (1995).

8. Harmand, J., Lobry, C., Rapaport, A., Sari, T.: The Chemostat: Mathematical Theory of Micro-organisms Cultures. Chemical Engineering Series, Wiley (2017).

9. Fekih-Salem, R., Harmand, J., Lobry, C., Rapaport, A., Sari, T.: Extensions of the chemostat model with flocculation. J. Math. Anal. Appl. 397, 292-306 (2013).

10. de Leenheer, P., Angeli, D., Sontag, E.:Crowding effects promote coexistence in the chemostat. J. Math. Anal. Appl. 319, 48-60 (2006).

11. Ricica, J.: Continuous cultivation of microorganisms, A review. Folia Microbiologica 16(5), 389-415 (1971).

12. Stanbury, P., Whitaker, A., Hall, S.: Principles of Fermentation Technology, $3^{\text {rd }}$ edition. Butterworth-Heinemann (2016).

13. Grady, L., Daigger, G., Lim, H.: Biological Wastewater Treatment, $3^{\text {rd }}$ edition. Environmental Science and Pollution Control Series. Marcel Dekker, New-York (1999).

14. Hutchinson, G.E.: A treatise on limnology. Vol. II: Introduction to lake biology and the limnoplankton. John Wiley \& Sons (1967).

15. Barlow, J., Schaffner, W., de Noyelles Jr., F., Peterson, B.: Continuous flow nutrient bioassays with natural phytoplankton populations.

In: Glass, G. (ed.): Bioassay Techniques and Environmental Chemistry. John Wiley \& Sons Ltd (1973).

16. Jannasch, H.W.: Steady state and the chemostat in ecology. Limnol. Oceanogr. 19(4), 716-720 (1974).

17. Kalff, J., Knoechel, R.: Phytoplankton and their Dynamics in Oligotrophic and Eutrophic Lakes. Annu. Rev. Ecol. Syst. 9, 475-495 (1978).

18. Rurangwa, E., Verdegem, M.C.J.: Microorganisms in recirculating aquaculture systems and their management. Rev. Aquacult. 7(2), $117-130$ (2015).

19. Creed, I., McKnight, D., Pellerin, B., Green, M., Bergamaschi, B., Aiken, G., Burns, D., Findlay, S., Shanley, J., Striegl, R., Aulenbach, B., Clow, D., Laudon, H., McGlynn, B., McGuire, K., Smith, R., Stackpoole, S.: The river as a chemostat: fresh perspectives on dissolved organic matter flowing down the river continuum. Can. J. Fish. Aquat. Sci. 72, 1272-1285 (2015). 
20. Kung, C.M., Baltzis, B.: The growth of pure and simple microbial competitors in a moving and distributed medium. Math. Biosci. 111, 295-313 (1992).

21. Dramé, A.: A semilinear parabolic boundary-value problem in bioreactors theory. Electron. J. Differ. Eq. 129, 1-13 (2004).

22. Nie, H., Wu, J.: Positive solutions of a competition model for two resources in the unstirred chemostat. J. Math. Anal. Appl 355, 231-242 (2009).

23. Smith, H., Tang, B., Waltman, P.: Competition in a n-vessel gradostat. SIAM J. Appl. Math. 91(5), 1451-1471 (1991).

24. Stephanopoulos, G., Fredrickson, A.: Effect of inhomogeneities on the coexistence of competing microbial populations. Biotechnol. Bioeng. 21, 1491-1498 (1979).

25. Haidar, I., Rapaport, A., Gérard, F.: Effects of spatial structure and diffusion on the performances of the chemostat. Math. Biosci. Eng. 8(4), 953-971 (2011).

26. Grieves, R.B., Pipes, W.O., Milbury, W.F., Wood, R.K.: Piston-flow reactor model for continuous industrial fermentations. J. Appl.

Chem. 14, 478-486 (1964).

27. Doran, P. Design of mixing systems for plant cell suspensions in stirred reactors. Biotechnol. Prog. 15, 319-335 (1999).

28. Dramé, A., Lobry, C., Harmand, J., Rapaport, A., Mazenc, F.: Multiple stable equilibrium profiles in tubular bioreactors. Math. Comp. Model. 48(11-12), 1840-1853 (2008).

29. Zambrano, J., Carlsson, B., Diehl, S.: Optimal steady-state design of zone volumes of bioreactors with Monod growth kinetics. Biochem.

Eng. J. 100, 59-66 (2015).

30. Diehl, S., Zambrano, J., Carlsson, B.: Steady-state analyses of activated sludge processes with plug-flow reactor. J. Environ. Chem. Eng. 5(1), 795-809 (2017).

31. Lovitt, R., Wimpenny, J.: The gradostat: A tool for investigating microbial growth and interactions in solute gradients. J. Gen. Microbiol. 6, 80 (1979).

32. Lovitt, R., Wimpenny, J.: The gradostat: a bidirectional compound chemostat and its applications in microbial research. J. Gen. Microbiol. 127, 261-268 (1981).

33. Tang, B.: Mathematical investigations of growth of microorganisms in the gradostat. J. Math. Biol. 23, 319-339 (1986).

34. Smith, H., Waltman, P.: The gradostat: A model of competition along a nutrient gradient. Microb. Ecol. 22, 207-226 (1991).

35. Jacquez, J.: Compartmental Analysis in Biology and Medicine, $3^{r d}$ edition. Ann Arbor, Michigan (1996).

36. Levenspiel, O.: Chemical reaction engineering, $3^{\text {rd }}$ edition. Wiley, New York (1999).

37. Fogler, S.: Elements of Chemical Reaction Engineering, $4^{\text {th }}$ edition. Prentice Hall, New-York (2008).

38. Hu, W., Wlashchin, K., Betenbaugh, M., Wurm, F., Seth, G., Zhou, W.: Cellular Bioprocess Technology, Fundamentals and Frontier. Lectures Notes, University of Minesota (2007).

39. Grobicki, A., Stuckey, D.: Hydrodynamic characteristics of the anaerobic baffled reactor. Water Res. 26, 371-378 (1992).

40. Roux, G., Dahhou, B., Queinnec, I.: Adaptive non-linear control of a continuous stirred tank bioreactor. J. Process Contr. 4(3), 121-126 (1994).

41. Roca, E., Ghommidh, C., Navarro, J.M., Lema, J.M.: Hydraulic model of a gas-lift bioreactor with flocculating yeast. Bioprocess Biosyst. Eng. 12(5), 269-272 (1995).

42. Valdes-Parada, F., Alvarez-Ramirez, J., Ochoa-Tapia, A.: An approximate solution for a transient two-phase stirred tank bioreactor with nonlinear kinetics. Biotechnol. Prog. 21(5), 1420-1428 (2005).

43. Saddoud, A., Sari, T., Rapaport, A., Lortie, R., Harmand, J., Dubreucq, E.: A mathematical study of an enzymatic hydrolysis of a cellulosic substrate in non homogeneous reactors. Proc. IFAC (CAB) (2010).

44. Nakaoka, S., Takeuchi, Y.: Competition in chemostat-type equations with two habitats. Math. Biosci. 201, 157-171 (2006).

45. Tsakiroglou, C., Ioannidis, M.: Dual-porosity modelling of the pore structure and transport properties of a contaminated soil. Eur. J. Soil Sci. 59(4), 744-761 (2008). 
46. Schwartz, R., Juo, A., McInnes, K.: Estimating parameters for a dual-porosity model to describe non-equilibrium, reactive transport in a fine-textured soil. J. Hydrol. 229(3-4), 149-167 (2000).

47. Lenas, P., Thomopoulos, N., Vayenas, D., Pavlou, S.: Oscillations of two competing microbial populations in configurations of two interconnected chemostats. Mathematical Biosciences, 148, 43-63 (1998).

48. Dong, X., Kojouharov H., Grover, J.: Mathematical models of nutrient recycling and toxin production in a gradostat. Computers \& Mathematics with Applications, 68(9), 972-985 (2014).

49. Tan, C., Wang, Y., Wu, H.: Population abundance of a two-patch chemostat system with asymmetric diffusion", journal. Journal of Theoretical Biology 474, 1-13 (2019).

50. Hayes, R.E., Mmbaga, J.P.: Introduction to Chemical Reactor Analysis, $2^{\text {nd }}$ edition. CRC Press (2012).

51. Nauman, E.: Chemical Reactor Design, Optimization, and Scaleup. McGraw-Hill Handbooks Series (2002).

52. Paul, E.L., Atiemo-Obeng, V.A., Kresta, S.M.: Handbook of Industrial Mixing: Science and Practice. Wiley (2004).

53. de Gooijer, C., Bakker, W., Beeftink, H., Tramper, J.: Bioreactors in series: an overview of design procedures and practical applications. Enzyme Microb. Technol. 18, 202-219 (1996).

54. Harmand, J., Rapaport, A., Trofino, A.: Optimal design of two interconnected bioreactors-some new results. AIChE J. 49(6), 1433-1450 (1999).

55. Harmand, J., Dochain, D.: The optimal design of two interconnected (bio)chemical reactors revisited. Comput. Chem. Eng. 30, 70-82 (2005).

56. Smith, H.: Monotone Dynamical Systems: An Introduction to the Theory of Competitive and Cooperative Systems. AMS Math. Surveys Monogr. 41 (1995).

57. Luyben, K., Tramper, J.: Optimal design for continuously stirred tank reactors in series using Michaelis-Menten kinetics. Biotechnol. Bioeng. 24, 1217-1220 (1982).

58. Nelson, M., Sidhu, H.: Evaluating the performance of a cascade of two bioreactors. Chem. Eng. Sci. 61, 3159-3166 (2006). 